\title{
W MASS AND WIDTH MEASUREMENTS AT LEP2
}

\section{Hugo Ruiz*}

IFAE, Edifici Cn, UAB. E-08193 Cerdanyola. Spain

E-mail: 'ruiz@ifae.es

ABSTRACT: The LEP2 program, operating at centre-of-mass energies above the $\mathrm{W}^{+} \mathrm{W}^{-}$ production threshold, has allowed for precision measurements of the $\mathrm{W}$ boson mass and width. Results are presented here for a total luminosity of about $2300 \mathrm{pb}^{-1}$, corresponding to the $91 \%$ of the data taken at LEP2. The most important sources of systematic uncertainty are described, focussing on some recent progress in their understanding and evaluation. A new analysis from the OpAL collaboration dedicated to extract the mass from the purely leptonic channel is also presented.

\section{Introduction}

The comparison of the direct measurement of $M_{\mathrm{W}}$ with the prediction from the rest of electroweak measurements provides an important test of self-consistency of the Standard Model. The measurement constraints also the mass of the Higgs boson $\left(M_{\mathrm{H}}\right)$ when it is combined with the direct measurement of $M_{\text {top }}$ at the Tevatron.

In the following sections, the measurements of $M_{\mathrm{W}}$ and $\Gamma_{\mathrm{W}}$ at LEP2 are described. Three stages are considered: event selection, event reconstruction and extraction of $M_{\mathrm{W}}$ and $\Gamma_{\mathrm{W}}$.

\section{Event selection}

$\mathrm{W}^{+} \mathrm{W}^{-}$events can be categorised in three different channels according to the final states: hadronic $\left(\mathrm{W}^{+} \mathrm{W}^{-} \rightarrow \mathrm{q} \overline{\mathrm{q}}^{\prime} \mathrm{q} \overline{\mathrm{q}}^{\prime}\right)$, semileptonic $\left(\mathrm{W}^{+} \mathrm{W}^{-} \rightarrow \mathrm{q} \overline{\mathrm{q}}^{\prime} \ell \bar{\nu}_{\ell}\right)$ and leptonic $\left(\mathrm{W}^{+} \mathrm{W}^{-} \rightarrow\right.$ $\left.\ell^{+} \nu_{\ell} \ell^{\prime-} \bar{\nu}_{\ell^{\prime}}\right)$. The production fractions are, respectively, $46 \%, 44 \%$ and $10 \%$.

The selection is adapted to the different signatures of each channel. Hadronic events are characterised by four jets and little missing energy. Semileptonic events contain two jets, one high-momentum lepton and high missing momentum. Leptonic events contain two high momentum leptons and large missing momentum.

${ }^{*}$ Speaker. 
The purity of the selection is as high as $99 \%$ for the semileptonic channel, $90 \%$ for the leptonic and $85 \%$ for the hadronic, the latest suffering mainly from qq contamination. The overall efficiencies are about $85 \%$ for the hadronic and semileptonic channels. For leptonic events the efficiency depends on the flavour of the lepton, and spans from $95 \%$ for muons to $60 \%$ for taus.

\section{Invariant mass reconstruction}

For the hadronic and semileptonic channels, the measurement of $M_{\mathrm{W}}$ and $\Gamma_{\mathrm{W}}$ is based on the reconstruction of the invariant mass $\left(m_{\mathrm{inv}}\right)$ of the $\mathrm{W}$ decay particles. In the second case, the missing energy and momentum are assumed to be carried by the neutrino. For the purely leptonic channel a complete kinematic reconstruction is not possible, and a dedicated analysis is described in section 6 .

For semileptonic and hadronic channels, the reconstruction is performed in three steps. First, the jets and leptons are clustered. Second, a kinematic fit is applied to the fourmomenta of jets and leptons imposing energy and momentum conservation from the initial state. This fit improves the resolution of the mass measurement by making use of the the precisely-known centre-of-mass energy of LEP. The third step applies only for the hadronic channel, and consists of pairing the jets that are more likely to come from the same W.

\section{Mass and width determination}

The underlying $M_{\mathrm{W}}$ and $\Gamma_{\mathrm{W}}$ are extracted from the distribution of $m_{\mathrm{inv}}$. The expected Breit-Wigner is convoluted with effects like hadronisation, detector response or ISR, that are difficult to describe in an analytical way. Different analyses have been designed to extract $M_{\mathrm{W}}$ taking these effects into account, yielding similar statistical precisions.

In the most widely used analysis (ALEPH, L3, OPAL), $M_{\mathrm{W}}$ is extracted by fitting the distribution of $m_{\mathrm{inv}}$ of data to MC predictions for several values of $M_{\mathrm{W}}$. Technically, it is not feasible to generate $\mathrm{MC}$ samples for a continuous-like set of values of $M_{\mathrm{W}}$, and therefore the corresponding distributions are obtained by re-weighting the events according to the ratio of $\mathrm{CC} 03$ matrix elements.

Another method (DELPHI, OPAL) constructs an event likelihood by convoluting a BreitWigner with a radiator function that accounts for ISR, a resolution function describing the detector effects and an estimation of the probability of the event being $\mathrm{W}^{+} \mathrm{W}^{-}$. A final bias correction based on fully-simulated MC events is applied at the end.

The measurement of $\Gamma_{\mathrm{W}}$ is performed using the same fitting tools, but allowing two free parameters in the fits: $M_{\mathrm{W}}$ and $\Gamma_{\mathrm{W}}$.

\section{Systematic uncertainties}

The extraction of $M_{\mathrm{W}}$ relies either explicitly (MC re-weighting) or implicitly (via bias corrections) on the agreement between data and MC. In fact, most of the sources of systematic uncertainty reflect the limited knowledge on some of the effects that are implemented in the MC. 
For instance, the systematic associated to the detector accounts for the lack of knowledge on the description of the detector response. The intensive calibrations and cross-checks performed with very high statistics in the clear environment of LEP1 allows the estimation of this effect to be at the level of $11 \mathrm{MeV}$.

The uncertainty due to photon emission effects (namely, ISR and FSR) is estimated by the comparison of different theoretical models, and yields an effect of $8 \mathrm{MeV}$.

There has been some recent developments in the evaluation and understanding of some systematic uncertainties, and hence they are described with some more detail in the following sub-sections.

\subsection{LEP Energy}

The uncertainty on the LEP energy $\left(E_{\mathrm{LEP}}\right)$ is propagated directly to the measurement of $M_{\mathrm{W}}$ through the kinematic fit. The average uncertainty on $E_{\mathrm{LEP}}$ is $20 \mathrm{MeV}$, and it scales to $17 \mathrm{MeV}$ on $M_{\mathrm{W}}$.

At LEP2, the energy was determined indirectly from the measurement of the total bending field. This method can only be calibrated in an absolute way for low (LEP1) energies, and therefore it relies on an extrapolation at LEP2. During the last two years of running, three methods were used to cross-check this extrapolation.

The first one, called energy-loss method, relied on the measurement of the frequency of the field provided to the beam to compensate for synchrotron radiation. The second method consisted in measuring the lepton bending angle after passing through a LEP dipole. The last one was based on the comparison of the radiative return peak with $M_{\mathrm{Z}}$. Results from the three analyses are compatible with the standard measurement, but do not contribute to any improvement on the uncertainty of the measurement of $E_{\mathrm{LEP}}$.

\subsection{Fragmentation}

Discrepancies between data and MC at the fragmentation (or hadronisation) stage can bias the measurement via bad reproduced association of particles to jets and via interplay with the detector (biases and non-linearities). This uncertainty has been estimated in the past by applying the analyses to MC samples with different fragmentation models (JETSET, HERWIG or ARIADNE), and taking the maximum difference between fitted masses. This difference has been recently reduced from $30 \mathrm{MeV}$ to around $10 \mathrm{MeV}$, due to the improved implementation of the hadronisation in the HERWIG model.

Conceptually, the comparison of MC models is hardly satisfactory, as the source of biases is the potential disagreement between data and MC. Two new approaches have been followed lately which try to estimate the fragmentation systematic from the comparison between data a MC.

The first approach tries to propagate to $M_{\mathrm{W}}$ the discrepancies on the fragmentationrelated variables by re-weighting techniques. In the second, fake $\mathrm{W}^{+} \mathrm{W}^{-}$events are built by merging and boosting $\mathrm{Z}$ events from LEP1. The huge statistics at the $\mathrm{Z}$ peak can be used to put a limit on the fragmentation systematic for the measurement of $M_{\mathrm{W}}$. The final estimation of the fragmentation systematic effect is $17 \mathrm{MeV}$, coming from the maximum effect observed from the different approaches. 


\subsection{Final state interactions}

FSI affects only the hadronic channel. In MC models, the hadronisation of the $\mathrm{W}$ bosons is assumed to occur independently, and therefore any correlation between them becomes a potential source of bias. Two QCD effects are expected to produce such correlations: Bose-Einstein (BE) and Colour Reconnection (CR).

Even though no rigorous theoretical description of this effects exist, a set of phenomenological models are available to estimate the systematic on $M_{\mathrm{W}}$. FSI uncertainty is considered to be correlated between the four experiments, and it deweights the contribution of this channel to $27 \%$ in the combination.

The origin of BE is the tendency of coherently-produced identical bosons to be close in phase space, that has been experimentally established in $\mathrm{Z}$ decays at LEP1. The models used by the LEP collaborations yield a $25 \mathrm{MeV}$ effect, but this is believed to be an overestimation. That is because analyses based on observation of particle correlations disfavour the MC implementation of these BE models.

CR refers to a re-arrangement of the colour flow between the decay chains of the Ws. As the separation of the decay vertices is small compared to the hadronic scale, the decay products from different Ws can overlap in space-time allowing the re-arrangement.

The available CR models predict effects on particle multiplicity, particle spectra and angular distributions relative to the four-jet topology. Analyses have been devoted to try to disfavour models by the comparison of such distributions on data and MC, but only very extreme models have been discarded. The effect on $M_{\mathrm{W}}$ coming from the surviving models is still of $40 \mathrm{MeV}$.

A new approach is being considered recently in the view of reducing the CR uncertainty. It consists of re-designing the analyses to make them less sensitive such effects. The modifications are based on dismissing information from these particles more potentially affected by CR: those in the inter-jet region and/or those with low momentum. There are good prospects of reducing the final error on $M_{\mathrm{W}}$ as well as the ratio between systematic and statistical uncertainties for the final publication.

\section{W mass from the purely leptonic channel}

In this channel, a complete kinematic reconstruction of the events is not possible. However, some properties of the event show sensitivity to $M_{\mathrm{W}}$. In a recent analysis (OPAL), the lepton momenta and the pseudo-mass distributions are used to extract $M_{\mathrm{W}}$. The pseudo-mass is obtained from the $\mathrm{W}^{+} \mathrm{W}^{-} \rightarrow \ell^{+} \nu_{\ell} \ell^{\prime-} \bar{\nu}_{\ell^{\prime}}$ events under an extra assumption that fully constraints the system: neutrinos are produced in the same plane as leptons.

This new analysis gives: $M_{\mathrm{W}}=81.43 \pm 0.42$ (stat) \pm 0.15 (syst) $\mathrm{GeV} / \mathrm{c}^{2}$.

\section{Results and conclusions}

The preliminary measurements of $M_{\mathrm{W}}$ at the four LEP experiments give compatible results of significant precision (see fig. $\underline{1}_{1}^{1}$ ), making the LEP combination the most accurate 


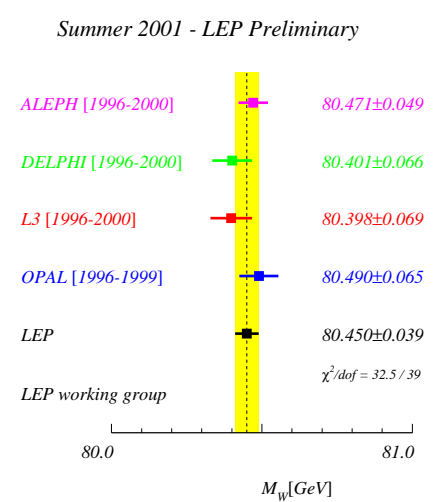

Figure 1: Combined measurement of $M_{\mathrm{W}}$ at LEP2 all channels.

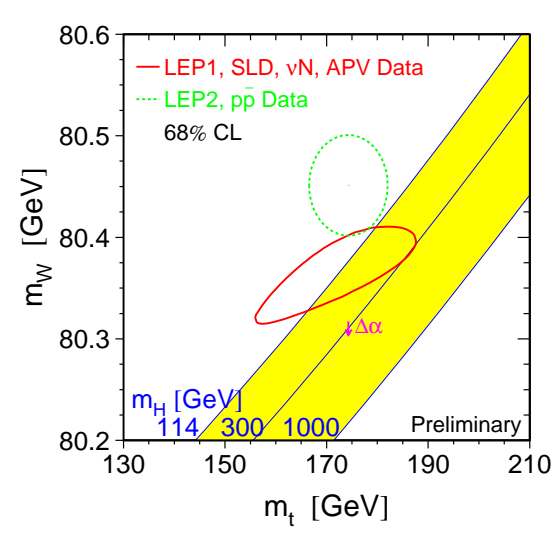

Figure 2: Comparison of direct measurements of $M_{\mathrm{W}}$ and $M_{\mathrm{top}}$ with SM predictions.

measurement of the W mass: $M_{\mathrm{W}}=80.450 \pm 0.039 \mathrm{GeV} / \mathrm{c}^{2}$. The combination with the measurement from the pp colliders gives the world average: $M_{\mathrm{W}}=80.451 \pm 0.033 \mathrm{GeV} / \mathrm{c}^{2}$.

This average can be compared with the prediction from a global fit to electroweak data assuming the SM: $M_{\mathrm{W}}=80.373 \pm 0.023 \mathrm{GeV} / \mathrm{c}^{2}$. This comparison is a stringent test of the SM. In figure $2_{i}^{-1}$ the direct measurements of $M_{\mathrm{W}}$ and $M_{\mathrm{top}}$ are compared with the predictions from the fits. Low values of $M_{\mathrm{H}}$ are favoured.

The difference between measurements from the semileptonic and the hadronic channel has been derived (assuming no FSI systematic), yielding $\Delta M_{\mathrm{W}}=7 \pm 41 \mathrm{Mev} / \mathrm{c}^{2}$. The small value sets an experimental limit on the impact of FSI effects on the measurement from the hadronic channel, of similar precision to the systematic uncertainty quoted for these effects $(47 \mathrm{MeV})$.

The combined LEP result for the $\mathrm{W}$ width measurement is: $\Gamma_{\mathrm{W}}=2.150 \pm 0.091 \mathrm{GeV} / \mathrm{c}^{2}$. The value is in good agreement with the $\mathrm{SM}$ prediction $\left(\Gamma_{\mathrm{W}}=2.09 \mathrm{GeV} / \mathrm{c}^{2}\right)$.

The effort put by the LEP collaborations in the understanding of some systematic effects anticipate a reduction of the uncertainty of the measurements for the final publication.

\section{Acknowledgments}

I would like to thank colleagues in the LEP W working group for the help in the preparation of this talk.

\section{References}

[1] LEP $\mathrm{W}^{+} \mathrm{W}^{-}$Working Group, combination for Summer 2001, http://lepewwg. web.cern.ch/LEPEWWG/lepww/mw/Summer01/ and references therein. 